\section{Visceral fat may increase prostate cancer risk}

Recent work by von Hafe et al. has shown a link between visceral fat and the risk of prostate cancer. Previous studies in this area have given conflicting results, possibly because visceral and subcutaneous fat accumulation have not been analyzed separately.

Von Hafe and colleagues from Portugal studied 63 patients with incident prostate cancer and 63 age-matched controls. Using a single CT scan of the abdomen at the level of the fourth lumbar vertebra, the investigators assessed the body fat distribution of each participant. Although the two groups were similar in terms of body-mass index, the mean area of abdominal fat was significantly higher in men with prostate cancer than in the control participants, mainly because of a higher mean area of visceral fat. Men whose ratio of visceral fat to subcutaneous fat lay in the upper tercile were more than 14 times more likely to have prostate cancer than those with lower ratios (odds ratio $14.5,95 \% \mathrm{Cl} 4.45-47.19)$. No relationship was seen between disease stage and body fat distribution.

The authors suggest that the observed relationship between visceral fat and prostate cancer risk might be associated with cytokines secreted by visceral fat cells, steroid hormone imbalances or increased insulin levels. As all the participants in this study were white, von Hafe et al. note that the results may not apply to other ethnic groups.

Original article von Hafe P et al. (2004) Visceral fat accumulation as a risk factor for prostate cancer. Obes Res 12: 1930-1935

\section{Role of KRAS in determining response to gefitinib or erlotinib}

Sensitivity to the small-molecule tyrosinekinase inhibitors gefitinib and erlotinib is associated with mutations in the EGFR gene. A recent study by $\mathrm{Pao}$ and colleagues has shown that mutations in KRAS, a member of the RAS family, appear to be linked to a lack of sensitivity to these drugs.

Sixty lung adenocarcinomas that were known to be sensitive or refractory to singleagent gefitinib or erlotinib were analyzed. Each tumor specimen was screened for mutations in EGFR exons 18-21 and KRAS exon 2, and mutation status was then compared with sensitivity to the two drugs.

KRAS mutations were found in $9(24 \%)$ of 38 tumors that were refractory to gefitinib or erlotinib, whereas none of these tumors bore EGFR mutations. Of 22 tumors sensitive to either drug, no KRAS mutations were found in the 21 specimens examined, but 17 (77\%) of 22 tumors bore EGFR mutations.

How to treat tumors with neither EGFR nor $K R A S$ mutations is still unclear. These data suggest, however, that KRAS mutations might predict a lack of response to gefitinib or erlotinib in patients with lung adenocarcinoma. Pao et al. conclude that it might be helpful to establish the mutational status of both EGFR and $K R A S$ when making treatment decisions in this setting, and they recommend that this be studied further in large, prospective trials.

Original article Pao W et al. (2005) KRAS mutations and primary resistance of lung adenocarcinomas to gefitinib or erlotinib. PLoS Medicine 2: 57-61

\section{Colorectal cancer and long-term meat consumption}

Red meat is thought to increase the risk of colorectal cancer, but questions remain regarding the magnitude of the association independent of other risk factors, and whether consumption of red or processed meat increases the risk of rectal cancer. In a recent paper published in JAMA, Chao et al. describe their investigation into effects of meat consumption on the risk of colon or rectal cancer in a large, population-based cohort in the US.

As part of the Cancer Prevention Study II, information on meat consumption was collected from a cohort of $\sim 150,000$ individuals in 1982, and again in 1992-1993. Participants were divided into three groups according to their meat intake (low, moderate or high).

During the follow-up period from 1992-1993 until 2001, there were 1,667 incident colorectal cancers. Adjusting for age and energy intake, a higher intake of red and processed meat in 1992-1993 was associated with a higher risk of colon cancer in both men and women. This association was no longer significant, however, when adjusted for other covariates 